\title{
Alternative Scenarios of Relativistic Heavy-Ion Collisions: III. Transverse Momentum Spectra
}

\author{
Yu.B. Ivanov ${ }^{1, \text { * }}$ \\ ${ }^{1}$ Kurchatov Institute, Moscow RU-123182, Russia
}

\begin{abstract}
Transverse-mass spectra, their inverse slopes and mean transverse masses in relativistic collisions of heavy nuclei are analyzed in a wide range of incident energies $2.7 \mathrm{GeV} \leq \sqrt{s_{N N}} \leq 39 \mathrm{GeV}$. The analysis is performed within the three-fluid model employing three different equations of state (EoS's): a purely hadronic EoS, an EoS with the first-order phase transition and that with a smooth crossover transition into deconfined state. Calculations show that inverse slopes and mean transverse masses of all the species (with the exception of antibaryons within the hadronic scenario) exhibit the step-like behavior similar to that observed for mesons and protons in available experimental data. This step-like behavior takes place for all considered EoS's and results from the freezeout dynamics rather than is a signal of the deconfinement transition. A good reproduction of experimental inverse slopes and mean transverse masses for light species (up to proton) is achieved within all the considered scenarios. The freeze-out parameters are precisely the same as those used for reproduction of particles yields in previous papers of this series. This became possible because the freeze-out stage is not completely equilibrium.
\end{abstract}

PACS numbers: 25.75.-q, 25.75.Nq, 24.10.Nz

Keywords: relativistic heavy-ion collisions, transverse spectra, hydrodynamics, onset of deconfinement

\section{INTRODUCTION}

This paper continues a series of reports on simulations of relativistic heavy-ion collisions within different scenarios [1 5]. These simulations were performed within a model of the three-fluid dynamics (3FD) 6] employing three different equations of state (EoS): a purely hadronic EoS 7] (hadr. EoS) and two versions of EoS involving the deconfinement transition [8]. These two versions are an EoS with the first-order phase transition (2-phase EoS) and that with a smooth crossover transition (crossover EoS). Details of these calculations are described in the first paper of this series [3] dedicated to analysis of the baryon stopping. The main questions addressed in these simulations are: Where and how does onset of deconfinement happen? What is the order of the deconfinement transition at high baryon densities?

In this paper I report results on transverse-mass spectra, incident-energy dependence of inverse slopes of these spectra and mean transverse masses in relativistic heavyion collisions in the energy range from $2.7 \mathrm{GeV}$ to 39 $\mathrm{GeV}$ in terms of center-of-mass energy $\left(\sqrt{s_{N N}}\right)$. This domain covers the energy range of the beam-energy-scan program at the Relativistic Heavy-Ion Collider (RHIC) at Brookhaven National Laboratory (BNL) and the low-energy-scan program at Super Proton Synchrotron (SPS) of the European Organization for Nuclear Research (CERN), energies of newly constructed Facility for Antiproton and Ion Research (FAIR) in Darmstadt and the Nuclotron-based Ion Collider Facility (NICA) in Dubna, as well as the Alternating Gradient Synchrotron (AGS) at BNL.

*e-mail: Y.Ivanov@gsi.de
Experimental data on transverse-mass $\left(m_{T}\right)$ spectra of charged kaons produced in central $\mathrm{Au}+\mathrm{Au}[9,10]$ and $\mathrm{Pb}+\mathrm{Pb}$ 11, 12] collisions exhibit a peculiar dependence on the incident energy. The inverse slope parameter of these spectra at mid-rapidity increases with incident energy in the AGS energy domain and then saturates at SPS energies. The inverse slope parameter depends on the transverse-mass interval of the exponential fit. The mean transverse mass provides an alternative measure of the $m_{T}$-spectra that is free of the above shortcoming. Excitation functions of the mean transverse mass manifest a similar step-like behavior for charged kaons and also pions and protons [11]. Such a behavior is not observed in proton-proton collisions.

In Refs. 13, 14 this step-like behavior was associated with onset of the deconfinement transition. This assumption was indirectly confirmed by the fact that microscopic transport models-the Hadron-String Dynamics (HSD), the Ultra-relativistic Quantum Molecular Dynamics 15] the Boltzmann-Uehling-Uhlenbeck (GiBUU) model [16] based on hadronic degrees of freedom, failed to reproduce the observed behavior of the kaon inverse slope 15, 16]. Later, when partonic degrees of freedom were included in the HSD model [17] (the Parton-Hadron-String Dynamics), the reproduction of the kaon inverse slopes became better. However, a good reproduction of all transversemass spectra within the GiBUU model was achieved by inclusion of three-body collisions in terms of hadronic degrees of freedom [18], i.e. by just enhancing the collisional interaction within the hadronic phase.

Hydrodynamic simulations of Ref. [19] succeeded to describe this step-like behavior. However, in order to reproduce it these hydrodynamic simulations required incident-energy dependence of the freeze-out temperature which almost repeated the shape of the corresponding kaon effective temperature. This happened even in 
spite of using an EoS involving the phase transition into quark-gluon plasma (QGP). This way, the problem of kaon effective temperatures was just translated into a problem of freeze-out temperatures. Moreover, results of Ref. [19] imply that peculiar incident-energy dependence of the kaon effective temperature may be associated with dynamics of freeze-out.

In Refs. 20, 21] it was shown that dynamical description of the freeze-out [22, 23], accepted in the 3FD model, naturally explains the step-like behavior of inverse-slope parameters even without any deconfinement transition in the EoS. This freeze-out dynamics, effectively resulting in a pattern similar to that of the dynamic liquid-gas transition, differs from conventionally used freeze-out schemes. This explanation is equally applicable to the energy dependence of the mean transverse mass. Later, within the Hybrid Hydro-Kinetic Model [24] it was confirmed that different freeze-out procedures have almost as much influence on the mean transverse mass excitation function as the EoS.

In this paper I demonstrate that inverse slopes and mean transverse masses of various species (with the exception of antibaryons within the hadronic scenario) exhibit step-like behavior for all considered EoS's. This is only the effect of the dynamical freeze-out [22, 23] accepted in the model. Moreover, the inverse slopes, mean transverse masses and particle yields are described within precisely the same freeze-out procedure, contrary to the common belief that the kinetic freeze-out should happen later that the chemical one. The reason why the unique freeze-out works both for kinetic and chemical quantities is described in the next section (sect. 【I).

As was demonstrated in the first papers of this series [1, 3, 4], onset of the deconfinement transition takes place in the region of top-AGS-low-SPS incident energies within the considered here first-order-transition and crossover scenarios. The experimental baryon stopping indicates certain signs of a deconfinement transition [1, 3] in this energy region. The hadronic scenario fails to reproduce antibaryon production [1, 3, 4] above this energy region, while the deconfinement scenarios do. The change of behavior of experimentally available excitation functions of inverse slopes and mean transverse masses also occurs in this energy range [25]. Therefore, in this paper the attention is primarily focused on this incident energy range. Before proceeding to discussion of inverse slopes and mean transverse masses it is reasonable to consider the transverse mass spectra themselves, which are the source data for the former ones.

\section{3FD MODEL}

A conventional way of applying the fluid dynamics to heavy-ion collisions at RHIC and LHC energies starts from an initial state that is prepared by means of various kinetic codes [28 31]. Such approaches disregard effects of a possible deconfinement transition at the stage of inter-penetration of colliding nuclei, and hence cannot be used for searching signals of deconfinement at this stage. Contrary to these approaches, the 3FD model treats the collision process from the very beginning, i.e. from the stage of cold nuclei up to freeze-out, within the fluid dynamics.

In order to take into account a finite stopping power at the initial stage of the nuclear collision, the 3FD model deals with two baryon-rich fluids which simulate a counter-streaming regime of leading baryon-rich matter initially associated with constituent nucleons of the projectile $(\mathrm{p})$ and target $(\mathrm{t})$ nuclei. In addition, newly produced particles, populating the mid-rapidity region, are associated with a fireball (f) fluid. Therefore, the 3-fluid approximation is a minimal way to simulate the finite stopping power at high incident energies. Each of these fluids is governed by conventional hydrodynamic equations which contain interaction terms in their right-hand sides. These interaction terms describe mutual friction of the fluids and production of the fireball fluid. In terms of the above-mentioned conventional applications of the one-fluid hydrodynamics, the friction results in production of an initial state for the fluid evolution, i.e. it gives rise to the intial equilibration of the colliding matter.

The friction between fluids was fitted to reproduce the baryon stopping observed in (net)proton rapidity distributions for each EoS, as it is described in Ref. [3] in detail. The baryon stopping turns out to be only moderately sensitive to the freeze-out energy density. The freeze-out energy density $\varepsilon_{\mathrm{f} r z}=0.4 \mathrm{GeV} / \mathrm{fm}^{3}$ was chosen mostly on the condition of the best reproduction of secondary particle yields.

It is important that the same freeze-out is used to describe both the chemical (particle abundances) and kinetic (energy and momentum spectra) observables. On the other hand, it is commonly accepted that chemical observables require higher freeze-out temperatures than the kinetic ones, that implies that the kinetic freeze-out occurs somewhat later that the chemical one. This is indeed true, if the system is assumed to be completely thermodynamically equilibrium at the freeze-out stage. However, it is not the case in the 3FD model.

In the 3FD model the baryon-rich fluids are either unified (i.e. mutually stopped and merged into a single unified fluid) or spatially separated to the instant of the freeze-out. However, the baryon-free f-fluid still keeps its identity. During the whole collision process, the interaction between the f-fluid and the baryon-rich ones causes their unification. Nevertheless, till the very freeze-out this unification is not complete. Therefore, the freezingout system consists of two overlapping fluids and hence, strictly speaking, is not thermodynamically equilibrium. Then, the temperatures occur to be low enough to reproduce the kinetic observables, while abundances of the secondary particles are enhanced by a contribution of the f-fluid to the extent that they also agree with data. If the unified baryon-rich fluid and the f-fluid are artificially unified into a single fluid, as it was done in Ref. [5], 


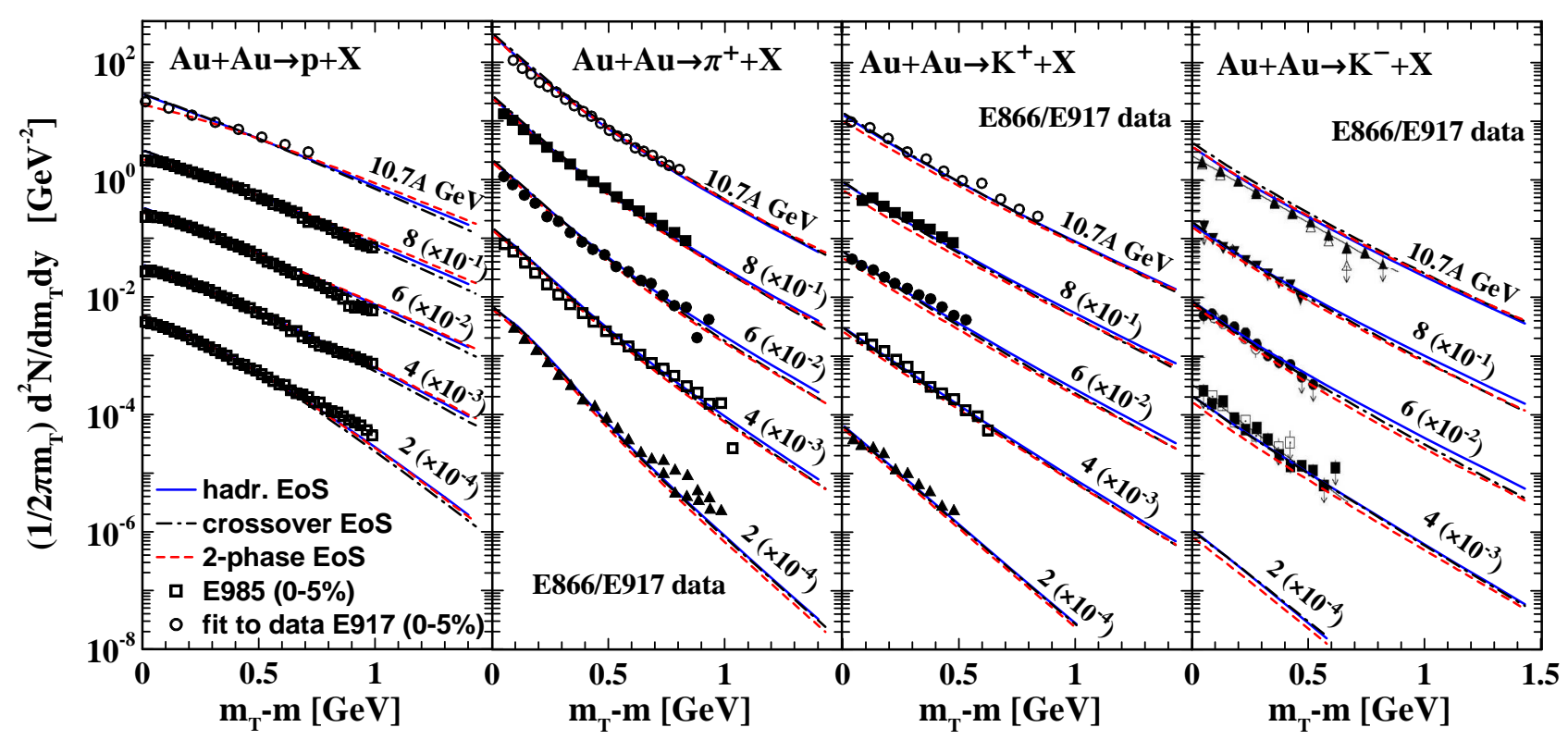

FIG. 1: (Color online) Transverse mass spectra (at midrapidity) of protons (data from E917 42] at 10.8A GeV and E895 43 ] at $2-8 \mathrm{~A} \mathrm{GeV}$ ), positive pions (data from E866 and E917 [9]), and positive (E866 and E917 [9]) and negative (E866 and E917 [10]) kaons from central collisions $\mathrm{Au}+\mathrm{Au}$ (5\% centrality) at AGS incident energies, $E_{\text {lab }}=2 A, 4 A, 6 A, 8 A$ and $10.7 A \mathrm{GeV}$, calculated with different EoS's at impact parameter $b=2 \mathrm{fm}$.

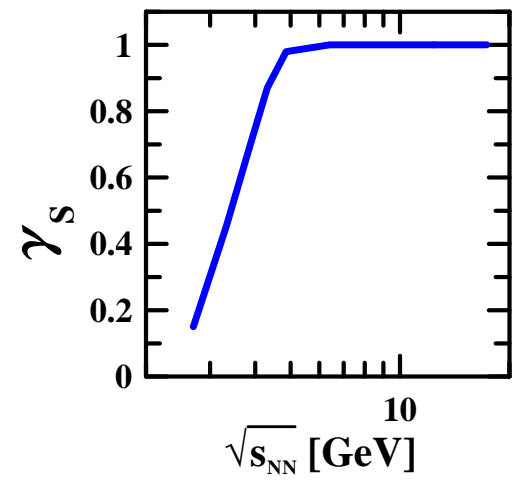

FIG. 2: (Color online) Strangeness suppression factor for central $\mathrm{Au}+\mathrm{Au}$ collisions as a function of the center-of-mass energy of colliding nuclei.

then the temperature and baryon chemical potential of this unified fluid well reproduce (within deconfinementtransition scenarios) the corresponding freeze-out parameters deduced from experimental data within the statistical model [26].

In Ref. [27] a comparison of results of transport (GiBUU) and hydrodynamic calculations for the expansion of a baryonrich hadronic fireball. Strong deviations from chemical equilibrium, especially at the final (freezeout) stage, were found within the transport calculations. This nonequilibrium results in an enhancement of yields of the secondary particles. Within the 3FD model the contribution of the f-fluid at the freeze-out precisely simulates this nonequilibrium enhancement.

The 3FD model [ 6 ] is a straightforward extension of the 2-fluid model with radiation of direct pions $32-34$ and (2+1)-fluid model 35, 36]. The above models were extend in such a way that the created baryon-free fluid (which is called a "fireball" fluid, following the Frankfurt group) is treated on equal footing with the baryonrich ones. In addition, a certain formation time $\tau$ is attributed to the fireball fluid, during which the matter of the fluid propagates without interactions. The formation time is associated with a finite time of string formation. It is similarly incorporated in kinetic transport models such as Ultrarelativistic Quantum Molecular Dynamics (UrQMD) [37] and HSD [38].

\section{TRANSVERSE MASS SPECTRA}

In this section I report results on transverse-mass $\left(m_{T}\right)$ spectra of various species from central $\mathrm{Au}+\mathrm{Au}$ (for AGS energies) and $\mathrm{Pb}+\mathrm{Pb}$ (for SPS energies) collisions. Correspondence between experimental centrality, i.e the fraction of the total reaction cross section related to a data set, and the mean value of the impact parameter $(b=$ $2.4 \mathrm{fm}$ for centra $\mathrm{Pb}+\mathrm{Pb}$ collisions) is taken from the paper [39] in case of NA49 data. For central Au+Au collisions the value of $b=2 \mathrm{fm}$ is approximately estimated proceeding from geometrical considerations. A contribution of weak decays of strange hyperons into non-strange hadron yields is disregarded in accordance with measurement conditions of the NA49 collaboration. At the AGS energies the contribution of weak decays is negligible. 3FD calculations for RHIC energies are not presented because the RHIC data for identified particles in the con- 


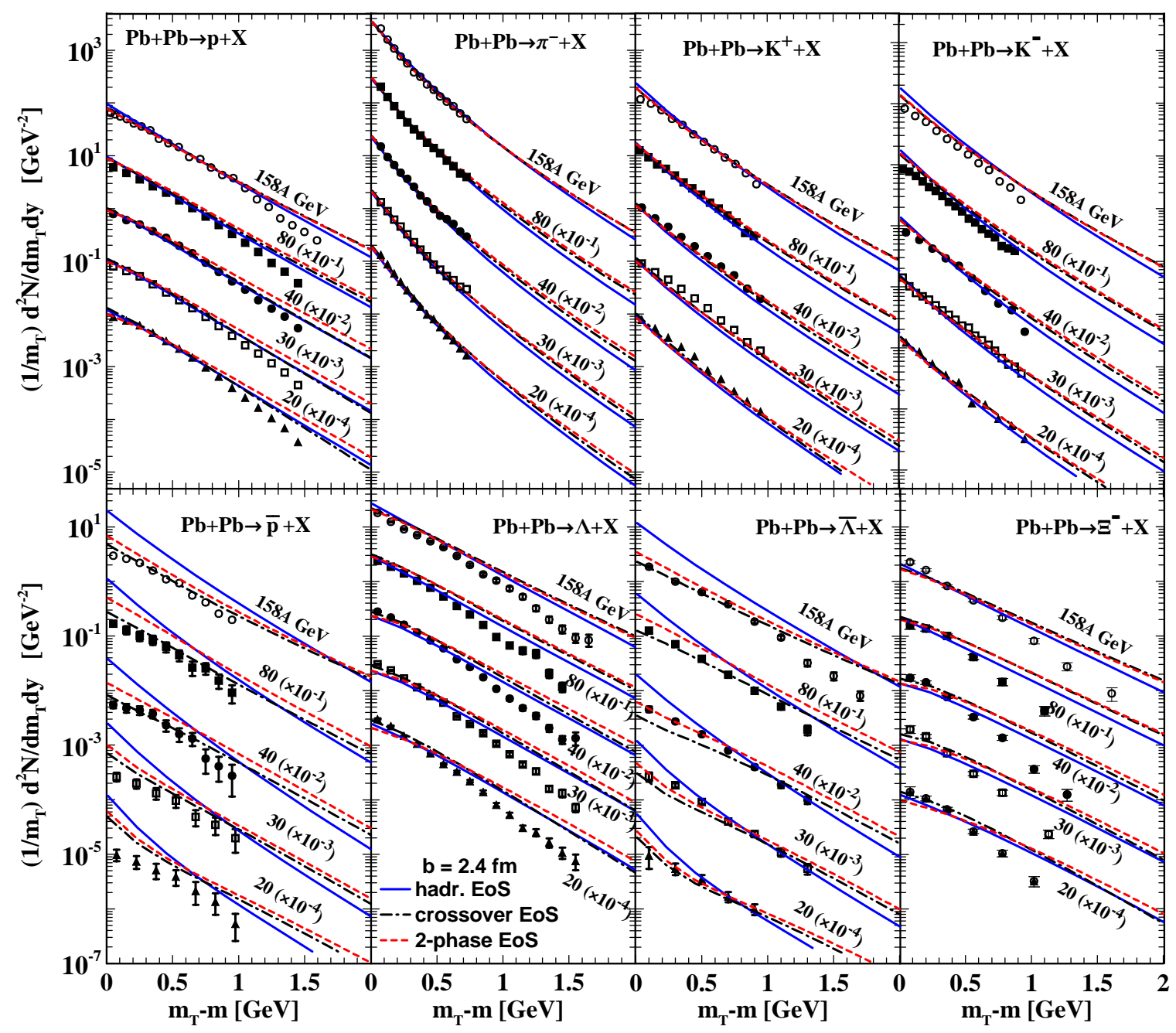

FIG. 3: (Color online) Transverse mass spectra (at midrapidity) of protons, pions, kaons, antiprotons, $\Lambda, \bar{\Lambda}$ and $\Xi^{-}$hyperons from central $\mathrm{Pb}+\mathrm{Pb}$ collisions $(b=2.4 \mathrm{fm})$ at SPS incident energies. Experimental data are from NA49 Collaboration 11 , 12, 45 49]. Calculations for $\Lambda, \bar{\Lambda}$ and $\Xi^{-}$hyperons at $E_{l a b}=158 \mathrm{~A} \mathrm{GeV}$ were performed at $b=4.6$ fm because the centrality selection of the respective data [49] is $0-10 \%$, contrary to other presented data corresponding to either $0-5 \%$ or $0-7 \%$ selection.

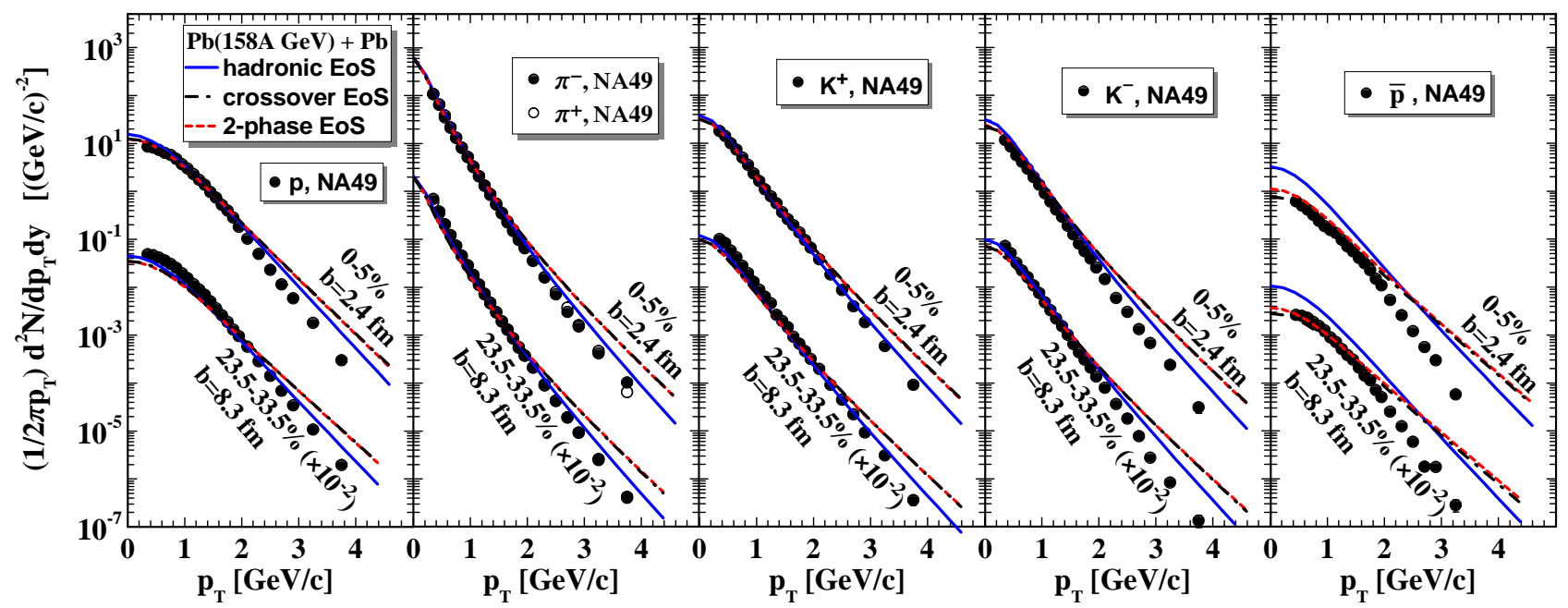

FIG. 4: (Color online) Transverse momentum spectra (at midrapidity) of protons, pions, kaons, and antiprotons from central collisions $\mathrm{Pb}+\mathrm{Pb}(b=2.4 \mathrm{fm})$ at $158 \mathrm{~A} \mathrm{GeV}$ in the extended $p_{T}$ range. Experimental data are from NA49 Collaboration [50]. 
sidered energy range are very fragmentary and/or have a preliminarily status [40, 41]. Therefore, predictions for the RHIC energies are done in terms of inverse slopes and mean transverse masses, see Sect. IV] At RHIC energies, contributions of weak decays of strange hyperons into non-strange hadron yields were included in accordance with measurement conditions of the STAR and PHENIX collaborations.

The $m_{T}$ spectra at mid-rapidity from central collisions at AGS energies are presented in Fig. 1. As seen, the experimental data are reasonably good reproduced within all considered scenarios. All these scenarios predict approximately the same results for all displayed species except for protons at $10 \mathrm{~A} \mathrm{GeV}$ within the 2-phase EoS. The 2-phase scenario better reproduces the shape of the proton spectrum at $10 \mathrm{~A} \mathrm{GeV}$, though somewhat underestimates its overall normalization which has been already discussed in Refs. [1, 3]. The strangeness production at low incident energies is overestimated within the $3 \mathrm{FD}$ model, since used EoS's are based on the grand canonical ensemble. Therefore, the spectra of single-strange particles, like $K^{ \pm}$, displayed in Fig. 1, are multiplied by $\gamma_{S}$ factor, that takes into account an additional strangeness suppression due to constraints of canonical ensemble [44]. The excitation function of the $\gamma_{S}$ factor is presented in Fig. 2, which is of course applicable only to central collisions of considered nuclei. As seen, at $E_{\text {lab }}>10 A \mathrm{GeV}$ there is no need for additional strangeness suppression.

The $m_{T}$ spectra at mid-rapidity from central collisions at SPS energies are presented in Fig. 3. As seen, for abundant probes (upper raw of panels) agreement with data is certainly better than that for rare ones (lower raw of panels). This is again an artifact of the description based based on grand-canonical statistics which requires "large" multiplicities to be valid. A lack of exact conservations (of baryon number and strangeness) in the grand-canonical ensemble results in overestimation of data for rare probes. The hadronic scenario fails to reproduce the antibaryon (antiproton and anti-lambda) spectra even at low $m_{T}$, as it has already been discussed in Ref. [4]. The 3FD predictions essentially overestimate the high- $m_{T}$ ends of these data. This is even better seen in comparison with the NA49 data [50] taken in a wide $p_{T}$ range at $158 \mathrm{~A} \mathrm{GeV}$, see Fig. 4. This overestimation is a manifestation of finiteness of the considered system. Even abundant hadronic probes become rare at high momenta. Therefore, their treatment on the basis of grand canonical ensemble results in overestimation of their yields. Moreover, the more rare probe is the hadron by itself, the stronger its high- $p_{T}$ end of the spectrum is suppressed due to restrictions of the canonical ensemble. In fact, the hadronic scenario closer reproduces the high- $m_{T}$ and $-p_{T}$ ends of the spectra as compared with the deconfinement-transition ones. However, this cannot be considered as an advantage of the hadronic scenario because the hydrodynamics is primarily expected to describe the soft parts of the spectra.

\section{INVERSE SLOPES OF $m_{T}$ SPECTRA AND MEAN TRANSVERSE MASSES}

In order to quantify the spectral shape, the invariant $m_{t}$ spectra are usually fitted by an exponential function

$$
\frac{d^{2} N}{m_{T} d m_{T} d y} \propto \exp \left(-\frac{m_{T}}{T(y)}\right),
$$

where $m_{T}$ and $y$ are the transverse mass and rapidity, respectively, and $T(y)$ is the inverse slope parameter that generally depends on the rapidity. Below we consider only slopes at midrapidity. Therefore, the argument $y$ is omitted. Incident energy dependence of inverse slope parameters at midrapidity for various species is shown in Fig. 5 .

The exponential function results in a very good fit for kaons at $m_{T}-m<1 \mathrm{GeV}$. For higher $m_{T}$ this fit underestimate the data, as it was demonstrated in Ref. [4] based on high- $p_{T}$ ends of these data [50]. Heavier particles exhibit deviation from an exponential behavior already at moderate $m_{T}$. Therefore, the fit results depend on the range in which the fit is performed. This range is indicated in each panel of Fig. 5. Nevertheless, inverse slope parameters $T$ extracted for different particles at different energies in a region up to moderately high $m_{T}$ is a useful tool to perform a spectacular comparison of different $m_{T}$ spectra.

The energy dependence of $T$ for charged kaons exhibits an interesting feature, as shown in Fig. 5. While $T$ is rapidly rising with center-of-mass energy for $\sqrt{s_{N N}}<$ $8 \mathrm{GeV}$, it is rather constant or only slightly increasing above this energy. A similar observation has been made for the mean transverse mass

$$
\left\langle m_{T}\right\rangle=\frac{\int d^{2} p_{T} m_{T}\left(\frac{d^{2} N}{m_{T} d m_{T} d y}\right)}{\int d^{2} p_{T}\left(\frac{d^{2} N}{m_{T} d m_{T} d y}\right)}
$$

of also pions and protons [12, 51], i.e. of those species experimental data for which are available below SPS energies, see Fig. 6] The mean transverse mass is a good measure of the transverse spectrum even if it is not exponential like in (1). In proton-proton collisions such a behavior is not observed 53]. Therefore, the step-like behavior of inverse slopes and mean transverse masses certainly results from collective motion of the matter.

Calculations within the 3FD model show that inverse slopes and mean transverse masses of all the species exhibit the same step-like behavior for all considered EoS's. The exception is the mean $\left\langle m_{T}\right\rangle$ of antibaryons within hadronic scenario, which fails to reproduce any antibaryon observables [4].

This step-like behavior is a consequence of the steplike behavior of the effective freeze-out energy density $\left\langle\varepsilon_{\text {out }}\right\rangle$, see Fig. 7. In fact, this explanation of the stepeffect is similar to that in hydrodynamic simulations of 


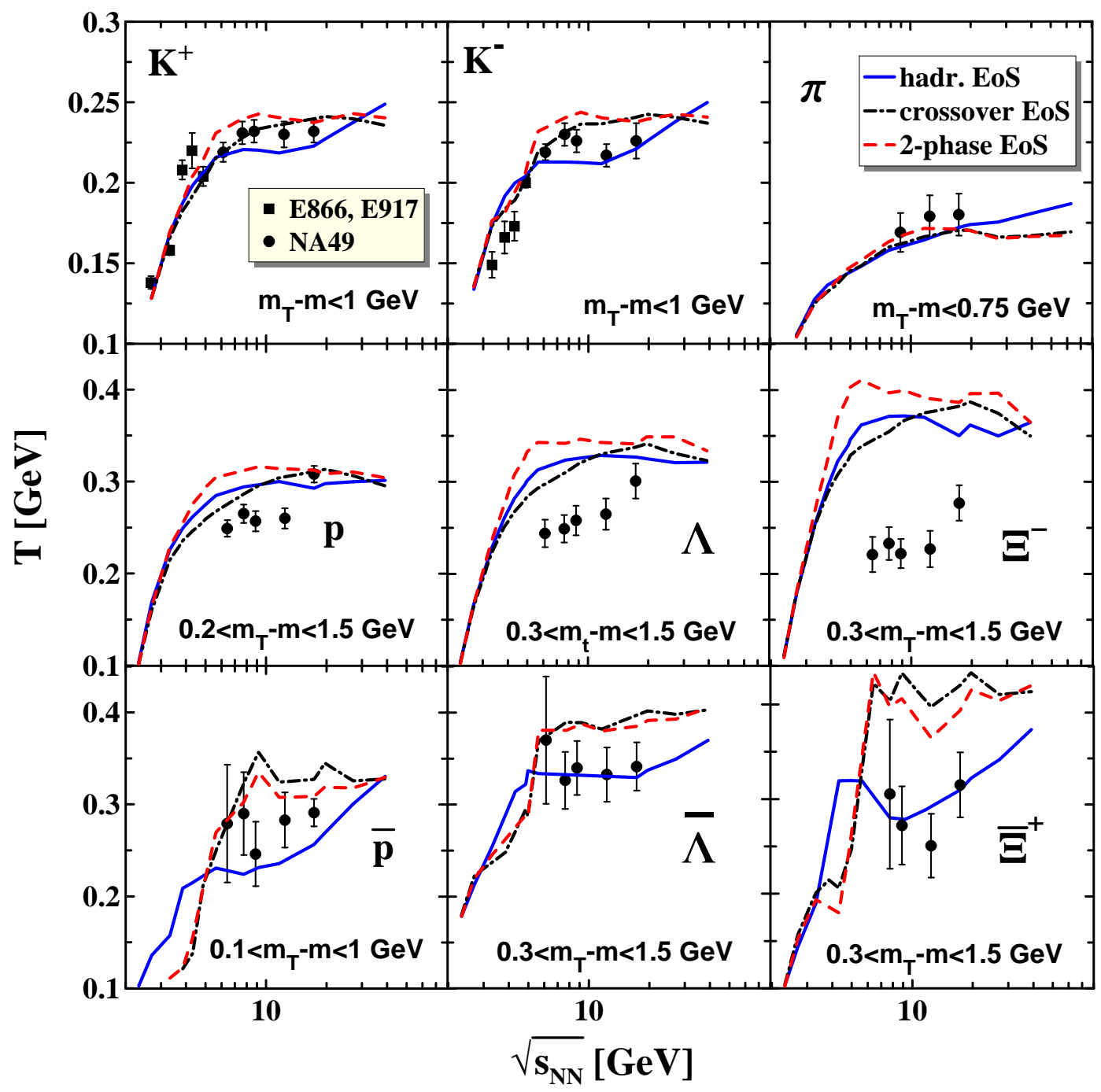

FIG. 5: (Color online) Inverse slopes of transverse-mass spectra (at midrapidity) of various species from central collisions $\mathrm{Au}+\mathrm{Au}$ (at AGS and RHIC energies, $b=2 \mathrm{fm}$ ) and $\mathrm{Pb}+\mathrm{Pb}$ (at SPS energies, $b=2.4 \mathrm{fm}$ ) as functions of the center-of-mass incident energy. Experimental data are taken from Refs. [12, 46, 49]. The $m_{T}$ range, in which the exponential fit (11) was performed, is indicated in each panel.

Ref. [19], where the step-like freeze-out temperature dependence on incident energy was required to reproduce the inverse-slope excitation fuctions of kaons. The 3FD model proceeds somewhat further as compared with Ref. 19] and explains the origin of this step-like behavior, however, not the height of the step itself that is still related to the value of the phenomenological parameterthe freeze-out energy density $\varepsilon_{\text {frz }}$. This parameter is the same for all EoS's and all incident energies ${ }^{1}: \varepsilon_{\text {frz }}=0.4$ $\mathrm{GeV} / \mathrm{fm}^{3}$. Contrary to the effective freeze-out energy density $\left\langle\varepsilon_{\text {out }}\right\rangle$ at which the freeze-out actually happens, the $\varepsilon_{\text {frz }}$ quantity has a meaning of a "trigger", that indicates possibility of the freeze-out.

\footnotetext{
${ }^{1}$ Only for the lowest considered incident energy of $2 A \mathrm{GeV}$ it was taken different: $0.3 \mathrm{GeV} / \mathrm{fm}^{3}$
}

The freeze-out procedure adopted in the 3FD model was analyzed in detail in Ref. 222. This method of freezeout can be called dynamical, since the freeze-out process here is integrated into the fluid dynamics through hydrodynamic equations. The freeze-out front is not defined just "geometrically" on the condition of the freeze-out criterion met ${ }^{2}$ but rather is a subject the fluid evolution. It competes with the fluid flow and not always reaches the place where the freeze-out criterion is first met. This kind of freeze-out is similar to the model of "continuous emission" proposed in Ref. [55] and further developed in Refs. [56, 57]. There the particle emission occurs from a surface layer of the mean-free-path width. In the 3FD

\footnotetext{
2 The freeze-out criterion demands that the energy density of the matter is lower than the value of $\varepsilon_{\mathrm{frz}}$.
} 


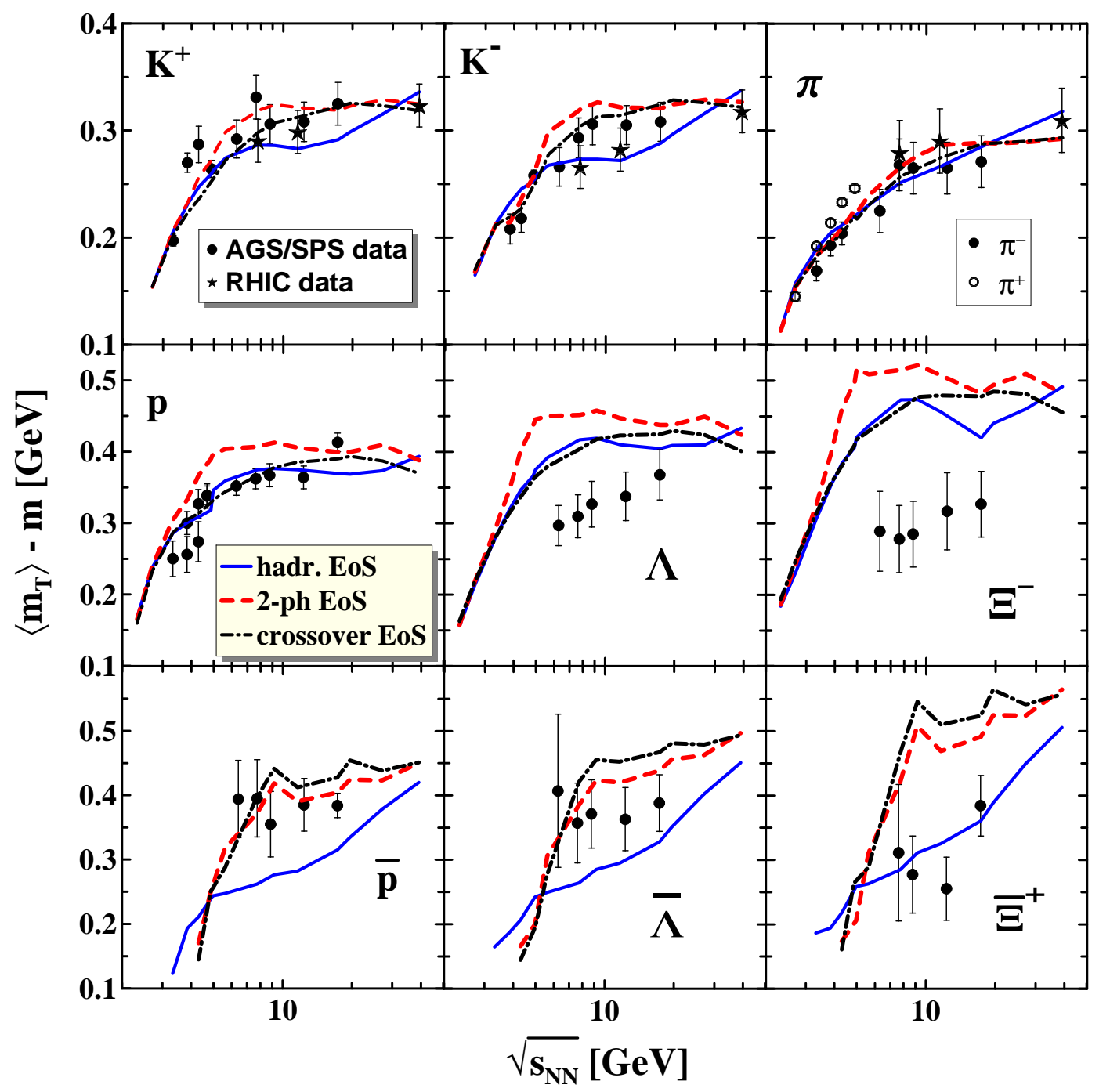

FIG. 6: (Color online) Mean transverse mass (minus particle mass) at midrapidity of various species from central collisions $\mathrm{Au}+\mathrm{Au}$ (at AGS and RHIC energies, $b=2 \mathrm{fm}$ ) and $\mathrm{Pb}+\mathrm{Pb}$ (at SPS energies, $b=2.4 \mathrm{fm}$ ) as functions of the center-of-mass incident energy. Experimental data are taken from Refs. [12, 46, 49, 51] for AGS-SPS energies and from Ref. [52] for RHIC energies.

model the physical pattern is similar, only the mean free path is shrunk to zero (in practice, to the width of the grid cell).

The physical pattern behind this freeze-out resembles the process of expansion of compressed and heated classical fluid into vacuum. Physics of this process is studied both experimentally and theoretically [58 62]. Evaporation from free surface of normal (not superheated) fluid is a very slow process. Accordingly, the freeze-out of matter of high density $\left(\varepsilon>\varepsilon_{\mathrm{frz}}\right)$ is suppressed in the 3FD model. During expansion the fluid becomes more rarefied, still remaining quite hot. Thus, the fluid becomes superheated at $\varepsilon<\varepsilon_{\text {frz }}$. It occurs first at the periphery of the system, which is first affected by the decompression wave. Evaporation from free surface of superheated fluid is already a fast process. Accordingly, the freeze-out is allowed, but not necessarily happens actually, at $\varepsilon<\varepsilon_{\text {frz }}$.
Situations are possible, when the freeze-out criterion is met in the whole slab near the free surface rather than only at the surface. Such situations are illustrated in [22]. Here we have a choice either to instantaneously freeze out this whole near-surface slab or to wait until the freezeout front will gradually traverse this slab (if ever). This choice is relied on results of experiments on evaporation from superheated fluids. It was shown (see, e.g., Ref. [61]) that the evaporation front propagates with respect to fluid not faster than with the speed of sound. Precisely this choice is realized in the 3FD model. Only matter in the surface layer gets frozen out and removed from the fluid evolution during a single time step, while inner parts of the matter keep on evolving hydrodynamically even in spite of meeting the the "trigger" freeze-out criterion. Thus, the matter can turn out to be over-rarefied to the instant of its freeze-out at the surface. This implies 


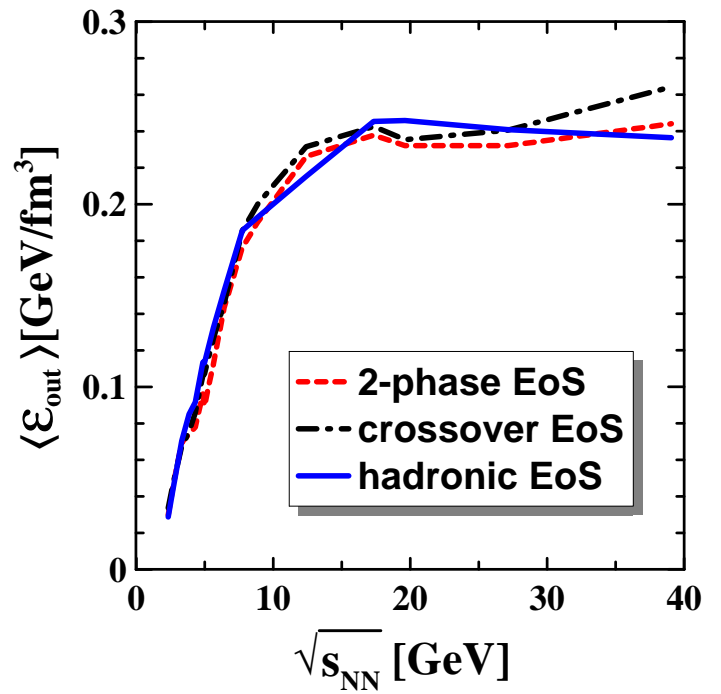

FIG. 7: (Color online) Mean energy density of the frozen-out matter as a function of incident energy.

that the freeze-out front may stay at essentially lower energy densities than $\varepsilon_{\text {frz }}$ because supersonic fluid expansion prevents it from reaching the region, where the condition $\varepsilon<\varepsilon_{\mathrm{frz}}$ is first met. This is precisely the case at low incident energies, as it was demonstrated in Ref. [22].

Physically it implies that a particle is evaporated ("frozen-out") only if it escapes from the system without collisions. Thus, its mean free path $\left(\lambda_{\mathrm{mfp}}\right)$ should be larger than its path to the free surface (with due account of the future evolution of the fluid). Precisely this criterion is applied in the model of "continuous emission" [55]. In the simplified 3FD version of the "continuous emission", $\lambda_{\mathrm{mfp}}=0$ (in practice, the cell width) in the fluid phase and $\lambda_{\mathrm{mfp}} \rightarrow \infty$ in the gas phase. Therefore, a particle can escape only from the free surface that cannot move inward the system faster than with the speed of sound [61].

The only exception from this rule is done at the final stage of the freeze-out. As it was observed in experiments with classical fluids (see, e.g., Ref. [59]), a fluid transforms into gas by explosion, if it is strongly superheated all over its volume. Therefore, at the final stage of the freeze-out, when criterion is met in the whole volume of the fluid residue, it is assumed that the whole residue becomes frozen out simultaneously. In particular, this is the reason why $\varepsilon_{\text {frz }}$ was chosen to be smaller for the lowest considered incident energy of $2 A \mathrm{GeV}: \varepsilon_{\text {frz }}\left(E_{l a b}=\right.$ $2 A \mathrm{GeV})=0.3 \mathrm{GeV} / \mathrm{fm}^{3}$. That was done because of a large contribution of the bulk freeze-out. In the energy range of $4-10 A \mathrm{GeV}$ the bulk freeze-out is not dominant and the freeze-out front does not reach the region, where $\varepsilon=\varepsilon_{\text {frz }}$. Hence, the 3 DF results in this energy range only weakly respond to variation of the freeze-out parameter $\varepsilon_{\text {frz }}$.

Of course, the freeze-out criterion, based on the energy density, is not universal. In particular, it is not applicable to the cold nuclear matter, which has $\varepsilon \approx 0.15 \mathrm{GeV} / \mathrm{fm}^{3}$ in its ground state. Therefore, the freeze-out procedure includes an additional condition, preserving the cold nuclear matted from being frozen out. It looks like this criterion is good enough for a restricted domain of the phase diagram, where freeze-out of hot nuclear matter really occurs.

The "step-like" behavior of $\left\langle\varepsilon_{\text {out }}\right\rangle$ (see Fig. 7) is a consequence of the freeze-out dynamics, as it was demonstrated in Ref. [22]. At low (AGS) incident energies, the energy density achieved at the border with vacuum, $\varepsilon^{s}$, is lower than $\varepsilon_{\text {frz }}$. Therefore, the surface freeze-out starts at lower energy densities. It further proceeds at lower densities up to the global freeze-out because the freeze-out front moves not faster than with the speed of sound, like any perturbation in the hydrodynamics. Hence it cannot overcome the supersonic barrier and reach dense regions inside the expanding system. With the incident energy rise the energy density achieved at the border with vacuum gradually reaches the value of $\varepsilon_{\text {frz }}$ and then even overshoot it. If the overshoot happens, the system first expands without freeze-out. The freeze-out starts only when $\varepsilon^{s}$ drops to the value of $\varepsilon_{\mathrm{frz}}$. Then the surface freeze-out occurs really at the value $\varepsilon^{s} \approx \varepsilon_{\text {frz }}$ and thus the actual freeze-out energy density saturates at the value $\left\langle\varepsilon_{\text {out }}\right\rangle \approx \varepsilon_{\text {frz }} / 2$, i.e. at the half fall from $\varepsilon^{s}$ to zero. This freeze-out dynamics is quite stable with respect to numerics 22].

It is convenient to discuss inverse slope parameters $T$ in terms of collective properties of the frozen out matter, i.e. in terms of the freeze-out temperature $T_{\text {frz }}$ and transverse velocity $v_{\mathrm{tr}}$. At moderate $v_{\mathrm{tr}}$, the relation between $T$ and $\left(T_{\text {frz }}, v_{\text {tr }}\right)$ approximately reads

$$
T \approx T_{\mathrm{frz}}+\frac{1}{2} m v_{\mathrm{tr}}^{2},
$$

where $m$ is the particle mass. This relation results from the nonrelativistic limit of the blast-wave model [63 65]. The freeze-out temperature $T_{\text {frz }}$ and transverse velocity $v_{\text {tr }}$ are assumed to be the same for all species, as they are collective quantities of the matter. Notice that the mean transverse mass is identical to the inverse slope parameter, it the spectrum is precisely of the exponential form (11). Therefore, all the reasoning below is equally applicable to the mean transverse masses.

Mean freeze-out temperatures $\left(\left\langle T_{f r z}\right\rangle\right)$ and transverse velocities $\left(\left\langle v_{t r}\right\rangle\right)$ of the baryon-rich and baryon-free fluids averaged over the frozen-out system are presented in Fig. 8 as functions of incident energy. The baryonrich fluids are either spatially separated or unified at the freeze-out stage. The baryon-free ("fireball") fluid remains undissolved in baryonic fluids till the freeze-out. As mentioned above, the fireball fluid is characterized by a certain formation time $\tau$, during which the matter of the fluid propagates without interactions. The main difference concerning this baryon-free fluid in considered alternative scenarios consists in different formation times: 
$\tau=2 \mathrm{fm} / \mathrm{c}$ for the hadronic scenario and $\tau=0.17 \mathrm{fm} / \mathrm{c}$ for scenarios involving the deconfinement transition [3].

As seen from simulations, the main contribution to baryon and meson yields comes from baryon-rich fluids. Only at highest considered energy of $\sqrt{s_{N N}}=39$ $\mathrm{GeV}$ approximately half of pions at the mid-rapidity are produced from the baryon-free fluid within the deconfinement-transition scenarios. For all other particles and considered energies this fraction is essentially lower. At the same time the fraction of half for pions at the mid-rapidity from the baryon-free fluid is achieved already at $\sqrt{s_{N N}} \simeq 9 \mathrm{GeV}$ (i.e. $E_{l a b} \simeq 40 \mathrm{~A} \mathrm{GeV}$ ) within the hadronic scenario. This is one of the reasons why $\tau$ was chosen so large in the hadronic scenario. Large formation time prevents absorption of the baryon-free matter by the baryon-rich fluids. Without this large contribution of the baryon-free fluid it is impossible to reproduce mesonic yields at SPS energies. However, this strongly developed baryon-free fluid makes bad job for antibaryons in the case of hadronic EoS. The reason is that antibaryons are dominantly produced from the baryon-free fluid even at lower considered incident energies. Their yields in the hadronic scenario strongly overestimate experimental data [4].

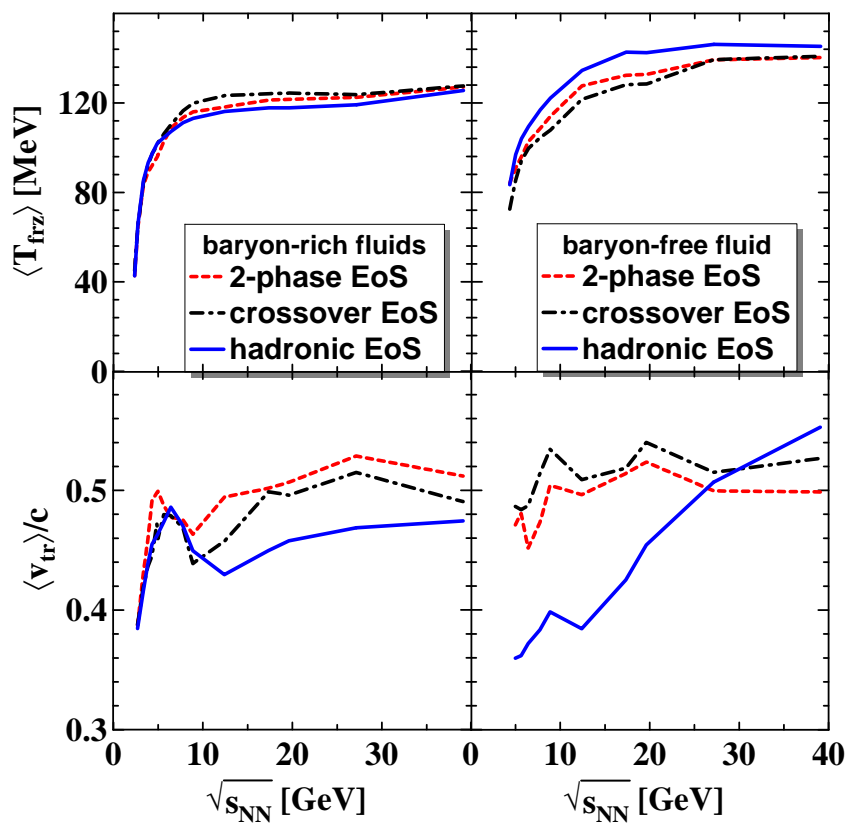

FIG. 8: (Color online) Mean temperatures $\left(\left\langle T_{f r z}\right\rangle\right)$ and transverse velocities $\left(\left\langle v_{t r}\right\rangle\right)$ of the frozen-out baryon-rich (left panels) and baryon-free fluids (right panels) averaged over the central layer of colliding system $(-2 \mathrm{fm} \leq z \leq 2 \mathrm{fm}$ with $z$ being the coordinate along the beam) as functions of incident energy.

The qualitative difference of excitation functions of mean transverse masses of antibaryons (lower panels in Fig. 6) within the hadronic scenario from those in the deconfinement-transition scenarios results from difference in dynamics of the baryon-free fluid. To a minor degree this qualitative difference also concerns the inverse slopes of antibaryons (lower panels in Fig. 5). To illustrate this in terms of quantities of the qualitative formula (3) let us consider mean temperatures $\left(\left\langle T_{f r z}\right\rangle\right)$ and transverse velocities $\left(\left\langle v_{t r}\right\rangle\right)$ of the frozen-out baryon-rich and baryon-free fluids averaged over the central region of the colliding system, see Fig. 8, The central region is chosen because the mid-rapidity region, in particular mid-rapidity transverse spectra, are predominantly populated by particles from the central spatial region. This central spatial region is defined as a layer orthogonal to the beam direction $(z)$ placed around origin of the $z$-axis, i.e. $-2 \mathrm{fm} \leq z \leq 2 \mathrm{fm}$.

As seen from Fig. 8, the mean freeze-out temperatures $\left(\left\langle T_{f r z}\right\rangle\right)$ of the baryon fluids exhibit a step-like behavior because of peculiarities of the freeze-out process discussed above. The temperatures only slightly rise above the "step" threshold. The transverse velocities $\left(\left\langle v_{t r}\right\rangle\right)$ of the baryon-rich fluids within all the considered scenarios exhibit very similar behavior. A dip in the range near $\sqrt{s_{N N}} \approx 10 \mathrm{GeV}$ is present in both hadronic and deconfinement-transition scenarios and is associated with transition from a single baryon-rich fireball at lower incident energies to two spatially separated fireballs at high energies. This is a point, where incomplete baryon stopping results in spatial separation of the projectile-like and target-like leading particles at the late stage of the evolution. It was illustrated in Ref. 22] for the hadronic scenario. For the deconfinement-transition scenarios the picture is similar. Therefore, as it results from Eq. (3), the inverse slopes and mean transverse masses of all the species, except for antibaryons, exhibit the step-like behavior with the above dip stronger or weaker revealed for different species.

The transverse velocities of the baryon-free fluid exhibit very different behavior for hadronic and deconfinement-transition scenarios. Whereas $\left\langle v_{t r}\right\rangle$ reveal saturation (up to numerical fluctuations) for deconfinement-transition scenarios, within the hadronic scenario the transverse velocity gradually grows with the incident energy rise. The formation time is short within deconfinement-transition scenarios. Hence, the baryonfree fluid is strongly coupled with the baryon-rich fluids almost from the instant of its birth. Therefore, its evolution is very similar to that of the baryon fluids. Based again on Eq. (3), we can expect the step-like behavior of the inverse slopes and mean transverse masses of antibaryons, as it is the case in Figs. [5 and 6, even numerical fluctuations are very similar.

The situation differs if the formation time is comparatively large like in the hadronic scenario. Therefore, the baryon-free fluid is less dragged by the baryon fluids and hence its transverse velocities turn out to be much less correlated with those of the baryon fluids. The baryonfree fluid evolves almost independently of the baryonrich fluids. Nevertheless, a weak remnant of the dip associated with transition from a single baryon-rich fireball at lower incident energies to two spatially separated 
fireballs at high energies is seen even in this case. For deconfinement-transition scenarios, this weak dip cannot be well distinguished against the background of the above mentioned numerical fluctuations. The increase of the transverse velocities with the incident energy rise results from growing density of the baryon-free fluid with the incident-energy rise. This behavior of the transverse velocities results in violation of the step-like behavior of mean transverse masses (and, to minor extent, of the inverse slopes) of antibaryons within the hadronic scenario. Indeed, the second term in Eq. (3) increases with the energy especially for large masses $m$ which is the case for antibaryons. Even inverse slopes and mean transverse masses of light mesons start to rise at high incident energies because of a large contributions of the baryon-free fluid to their yields.

The inverse slope parameters deduced from experiment [54] increase linearly with particle mass up to the mass of $\Lambda$ and $\bar{\Lambda}$ hyperons, see Fig. 5. Though, already for the $\Lambda$ hyperon this dependence is slightly violated. This can be understood as a consequence of the radial expansion of the fireball, which, in a simplified picture, is described by Eq. (3). The same dependence on mass takes place for the mean transverse masses, see Fig. 6. Naturally, the same approximately linear dependence of the inverse slope on the particle mass is predicted by 3FD calculations.

However, the data on inverse slopes of heavy hadrons beginning from the mass of $\Xi^{-}$and $\Xi^{+}$hyperons do not fit into this systematics. In Ref. 54 it was conjectured that the heavy strange particles do not participate in the radial flow to the same extent as light particles. The suggested in Ref. [54] interpretation of this behavior is based on the assumption that rare heavy particles have a lower hadronic scattering cross section than light hadrons and therefore do not participate in the radial flow that is developing during the hadronic phase of the fireball evolution. This leads to the conclusion that a substantial part of the transverse expansion probed by these particles has to be generated during the partonic phase. Thus, the rare heavy particles could be directly sensitive to the pressure in the early phase of the reaction.

However, a more plausible interpretation of the violation of the linear $m$-dependence is possible. As it was mentioned in the previous section, rare particles are additionally suppressed due to restrictions of the canonical ensemble. Moreover, the more rare probe is the hadron by itself, the stronger its high- $p_{T}$ end of the spectrum is suppressed due to restrictions of the canonical ensemble. This high- $p_{T}$-enhanced suppression results in steeper slopes of the $m_{T}$ spectra than these would be in the grand canonical ensemble, i.e. in a large system. This, in its turn, manifests itself in a lower $T$ than it would be expected from the linear law of Eq. (3).

\section{SUMMARY}

Results on transverse-mass spectra in relativistic heavy-ion collisions in the energy range from $2.7 \mathrm{GeV}$ to $39 \mathrm{GeV}$ in terms of center-of-mass energy, $\sqrt{s_{N N}}$, are presented. These simulations were performed within the 3FD model [6] employing three different EoS's: a purely hadronic EoS [7], and two versions of EoS involving the deconfinement transition [8]. These two versions are an EoS with the first-order phase transition and that with a smooth crossover transition. Details of these calculations are described in the first paper of this series [3] dedicated to analysis of the baryon stopping.

If was found that within all scenarios the available data on $m_{T}$-spectra are reproduced approximately to the same extent almost for all hadronic species (with the exception of antibaryons within the hadronic scenario) in the AGSSPS energy range, i.e. from $2.7 \mathrm{GeV}$ to $17.4 \mathrm{GeV}$ in terms of $\sqrt{s_{N N}}$. The reproduction is better for abundant species and at low transverse masses. This a natural result of the fact that the model is based on grandcanonical statistics which require high multiplicities of species to be valid. The grand-canonical statistics overestimates production of rare species because it does not take onto account restrictions imposed by exact conservations (of strangeness, baryon charge, energy) in a finite system. Even abundant hadronic probes become rare at high momenta. Therefore, their treatment on the basis of grand canonical ensemble results to overestimation of their yield. Moreover, the more rare probe is by itself, the stronger its high- $m_{T}$ end of the spectrum is suppressed due to restrictions of the canonical ensemble.

In the case of hadronic EoS this agreement is achieved at the expense of noticeable enhancement the inter-fluid friction in the hadronic phase [3, 6] as compared with its microscopic estimate of Ref. [66]. However, thus tuned hadronic scenario fails to describe $m_{T}$-spectra of antibaryons even at low transverse masses. In fact, this result was expected in view of the earlier reported failure to reproduce antibaryon rapidity distributions within the hadronic scenario [4]. The advantage of deconfinementtransition scenarios is that they reproduce (with all the above-mentioned constraints) $m_{T}$-spectra of all species, including antibaryons, and do not require for that any modification of the microscopic friction in the hadronic phase.

Excitation functions of inverse slope parameters of $m_{T^{-}}$ spectra of various hadrons and their mean transverse masses at mid-rapidity were calculated in the $\sqrt{s_{N N}}$ range from $2.7 \mathrm{GeV}$ to $39 \mathrm{GeV}$. Calculations within the 3 FD model show that inverse slopes and mean transverse masses of all the species (with the exception of antibaryons within the hadronic scenario) exhibit the steplike behavior similar to that observed in experimental data. The exception is the mean $\left\langle m_{T}\right\rangle$ of antibaryons within hadronic scenario, which fails to reproduce any antibaryon observables [4].

This step-like behavior takes place for all considered 
EoS's and hence is not a signal of the deconfinement transition. This behavior is a consequence of the steplike behavior of the effective freeze-out energy density $\varepsilon_{\text {out }}$ unlike the phenomenological parameter-the freezeout energy density $\varepsilon_{\text {frz }}$ which remains constant for all considered incident energies and has a meaning of a "trigger", which indicate possibility of the freeze-out. The dynamics of the freeze-out process incorporated into the $3 F D$ model allows to explain how this "trigger" $\varepsilon_{\text {frz }}$ value gives rise to the step-like behavior of the effective $\varepsilon_{\text {out }}$. However, the nature of the "trigger" $\varepsilon_{\text {frz }}$ value still has no explanation and serves as a purely phenomenological parameter. In fact, similar explanation of the step-effect was indirectly implied in hydrodynamic simulations of Ref. [19], where the step-like freeze-out temperature dependence on incident energy was required to reproduce the inverse-slope excitation fuctions of kaons. The 3FD model goes somewhat further as compared with Ref. [19] by explaining the origin of this step-like behavior.

Quantitative agreement with experimental data on inverse slopes and mean transverse masses is achieved for "abundant" species, i.e. pions, kaons, protons, antiprotons and even anti-Lamdas. It is still surprising that the
$\Lambda$ hyperon does not enter this list. However, data on rare probes like $\Xi^{-}$and $\bar{\Xi}^{+}$hyperons turn out considerably lower than predictions of the 3FD model and even than data on lighter particles like $p, \bar{p}, \Lambda$ and $\bar{\Lambda}$. It is argued that this is a consequence of additional suppression due to restrictions of the canonical ensemble, which are not taken into account in the $3 \mathrm{FD}$ calculations.

All this indicates that a deconfinement-transition scenarios are certainly preferable in dscribing available data in the energy range from $2.7 \mathrm{GeV}$ to $39 \mathrm{GeV}$. This conclusion agrees with those deduced in the previous papers of this series [1, 3, 4].

\section{Acknowledgements}

I am grateful to A.S. Khvorostukhin, V.V. Skokov, and V.D. Toneev for providing me with the tabulated 2-phase and crossover EoS's. The calculations were performed at the computer cluster of GSI (Darmstadt). This work was supported by The Foundation for Internet Development (Moscow) and also partially supported by grant NS-215.2012.2.
[1] Yu. B. Ivanov, Phys. Lett. B 721, 123 (2013).

[2] Yu. B. Ivanov, Phys. Lett. B 723, 475 (2013).

[3] Yu. B. Ivanov, Phys. Rev. C 87, 064904 (2013).

[4] Yu. B. Ivanov, Phys. Rev. C 87, 064905 (2013).

[5] Yu. B. Ivanov, Phys. Lett. B 726, 422 (2013).

[6] Yu. B. Ivanov, V. N. Russkikh, and V.D. Toneev, Phys. Rev. C 73, 044904 (2006).

[7] V. M. Galitsky and I. N. Mishustin, Sov. J. Nucl. Phys. 29, 181 (1979).

[8] A. S. Khvorostukhin, V. V. Skokov, K. Redlich, and V. D. Toneev, Eur. Phys. J. C48, 531 (2006).

[9] L. Ahle et al. [E866 and E917 Collaborations], Phys. Lett. B 476, 1 (2000).

[10] L. Ahle et al. [E866 and E917 Collaborations], Phys. Lett. B 490, 53 (2000).

[11] S. V. Afanasiev et al. [NA49 Collaboration], Phys. Rev. C 66, 054902 (2002).

[12] C. Alt et al. [NA49 Collaboration], Phys. Rev. C 77, 024903 (2008).

[13] M.I. Gorenstein, M. Gazdzicki, and K. Bugaev, Phys. Lett. B567, 175 (2003).

[14] B. Mohanty, J. Alam, S. Sarkar, T.K. Nayak, B.K. Nandi, Phys. Rev. C 68, 021901 (2003).

[15] M. Bleicher, E. Bratkovskaya, S. Vogel, and X. Zhu, J. Phys. G31, S709 (2005); E.L. Bratkovskaya, M. Bleicher, M. Reiter, S. Soff, H. Stoecker, M. van Leeuwen, S. Bass, and W. Cassing, Phys. Rev. C 69, 054907 (2004); E.L. Bratkovskaya, S. Soff, H. Stoecker, M. van Leeuwen, and W. Cassing, Phys. Rev. Lett. 92, 032302 (2004).

[16] M. Wagner, A.B. Larionov, and U. Mosel, Phys. Rev. C 71 (2005) 034910.

[17] W. Cassing and E. L. Bratkovskaya, Nucl. Phys. A831, 215 (2009).

[18] A.B. Larionov, O. Buss, K. Gallmeister, and U. Mosel,
Phys. Rev. C 76, 044909 (2007).

[19] M. Gazdzicki, M.I. Gorenstein, F. Grassi, Y. Hama, T. Kodama, and O. Socolowski Jr, Braz. J. Phys. 34, 322 (2004).

[20] Yu. B. Ivanov and V. N. Russkikh, Eur. Phys. J. A 37, 139 (2008).

[21] Yu. B. Ivanov and V. N. Russkikh, Phys. Rev. C 78, 064902 (2008).

[22] V. N. Russkikh and Y. B. Ivanov, Phys. Rev. C 76, 054907 (2007).

[23] Y. B. Ivanov and V. N. Russkikh, Phys. Atom. Nucl. 72, 1238 (2009).

[24] H. Petersen, J. Steinheimer, M. Bleicher and H. Stocker, J. Phys. G 36, 055104 (2009).

[25] M. Gazdzicki, M. Gorenstein and P. Seyboth, Acta Phys. Polon. B 42, 307 (2011).

[26] A. Andronic, P. Braun-Munzinger and J. Stachel, Phys. Lett. B 673, 142 (2009) [Erratum-ibid. B 678, 516 (2009)].

[27] L. M. Satarov, A. B. Larionov and I. N. Mishustin, Phys. Rev. C 85, 054910 (2012).

[28] H. Petersen, J. Steinheimer, G. Burau, M. Bleicher, and H. Stocker, Phys. Rev. C 78, 044901 (2008).

[29] J. Steinheimer V. Dexheimer, H. Petersen, M. Bleicher, S. Schramm, and H. Stoecker, Phys. Rev. C 81, 044913 (2010).

[30] Y. Hama, T. Kodama and O. Socolowski, Jr., Braz. J. Phys. 35, 24 (2005).

[31] C. Nonaka and M. Asakawa, PTEP 2012, 01A208 (2012).

[32] I.N. Mishustin, V.N. Russkikh, and L.M. Satarov, Yad. Fiz. 48, 711 (1988) [Sov. J. Nucl. Phys. 48, 454 (1988)].

[33] V. N. Russkikh, Yu. B. Ivanov, Yu. E. Pokrovsky, and P. A. Henning, Nucl. Phys. A572, 749 (1994). 
[34] I.N. Mishustin, V.N. Russkikh, and L.M. Satarov, Yad. Fiz. 54, 429 (1991) [Sov. J. Nucl. Phys. 54, 260 (1991)].

[35] U. Katscher, D.H. Rischke, J.A. Maruhn, W. Greiner, I.N. Mishustin, and L.M. Satarov, Z. Phys. A346, 209 (1993); A. Dumitru, U. Katscher, J.A. Maruhn, H. Stöcker, W. Greiner, and D.H. Rischke, Phys. Rev. C 51, 2166 (1995); Z. Phys. A353, 187 (1995).

[36] J. Brachmann, A. Dumitru, J.A. Maruhn, H. Stöcker, W. Greiner, and D.H. Rischke, Nucl. Phys. A619, 391 (1997); M. Reiter, A. Dumitru, J. Brachmann, J.A. Maruhn, H. Stöcker, and W. Greiner, Nucl. Phys. A643, 99 (1998); M. Bleicher, M. Reiter, A. Dumitru, J. Brachmann, C. Spieles, S.A. Bass, H. Stöcker, and W. Greiner, Phys. Rev. C 59, R1844 (1999); J. Brachmann, A. Dumitru, H. Stöcker, and W. Greiner, Eur. Phys. J. A8, 549 (2000).

[37] S. Bass, et al., Prog. Part. Nucl. Phys. 41, 225 (1998).

[38] W. Cassing and E. L. Bratkovskaya, Phys. Rept. 308, 65 (1999).

[39] C. Alt et al. [NA49 Collaboration], Phys. Rev. C 68, 034903 (2003).

[40] X. Zhu [STAR Collaboration], Acta Phys. Polon. Supp. 5, 213 (2012).

[41] B. I. Abelev et al. [STAR Collaboration], Phys. Rev. C 81, 024911 (2010).

[42] B. B. Back, R. R. Betts, J. Chang, W. C. Chang, C. Y. Chi, Y. Y. Chu, J. B. Cumming and J. C. Dunlop et al., Phys. Rev. C 66, 054901 (2002).

[43] J. L. Klay et al. [E895 Collaboration], Phys. Rev. Lett. 88, 102301 (2002).

[44] P. Koch, B. Muller and J. Rafelski, Phys. Rept. 142, 167 (1986).

[45] M. Gazdzicki et al. [NA49 Collaboration], J. Phys. G 30, S701 (2004).

[46] C. Alt et al. [NA49 Collaboration], Phys. Rev. C 73, 044910 (2006).

[47] T. Anticic et al. [NA49 Collaboration], Phys. Rev. C 69, 024902 (2004).

[48] T. Anticic et al. [NA49 Collaboration], Phys. Rev. Lett.
93, 022302 (2004).

[49] C. Alt et al. [NA49 Collaboration], Phys. Rev. C 78, 034918 (2008).

[50] C. Alt et al. [NA49 Collaboration], Phys. Rev. C 77, 034906 (2008).

[51] C. Blume [NA49 Collaboration], J. Phys. G 31, S685 (2005).

[52] L. Kumar [STAR Collaboration], J. Phys. G 38, 124145 (2011).

[53] M. Kliemant, B. Lungwitz and M. Gazdzicki, Phys. Rev. C 69, 044903 (2004).

[54] C. Blume and C. Markert, Prog. Part. Nucl. Phys. 66, 834 (2011).

[55] F. Grassi, Y. Hama, and T. Kodama, Phys. Lett. B355, 9 (1995); Z. Phys. C73, 153 (1996); Yu.M. Sinyukov, S.V. Akkelin, and Y. Hama, Phys. Rev. Lett. 89, 052301 (2002); F. Grassi, Braz. J. Phys. 35, 52 (2005).

[56] S.V. Akkelin, Y. Hama, Iu.A. Karpenko, and Yu.M. Sinyukov, Phys. Rev. C 78, 034906 (2008).

[57] J. Knoll, Nucl. Phys. A 821, 235 (2009).

[58] C.J. Knight, J. Fluid Mech. 75, 469 (1976).

[59] J.E. Shepherd and B. Sturtevant, J. Fluid Mech. 121, 379 (1982).

[60] Th. Kurschat, H. Chaves and G.E.A. Meier J. Fluid Mech. 236, 43 (1992).

[61] J.R. Simoes-Moreira and J.E. Shepherd, J. Fluid Mech. 382, 63 (1999).

[62] O. Le Metayer, J. Massoni, and R. Saurel, J. Comp. Phys. 205, 567 (2005).

[63] J. P. Bondorf, S. I. A. Garpman and J. Zimanyi, Nucl. Phys. A296, 320 (1978).

[64] P. J. Siemens and J. O. Rasmussen, Phys. Rev. Lett. 42, 880 (1979).

[65] E. Schnedermann, J. Sollfrank and U. W. Heinz, Phys. Rev. C 48, 2462 (1993)

[66] L.M. Satarov, Yad. Fiz. 52, 412 (1990) [Sov. J. Nucl. Phys. 52, 264 (1990)]. 